$116 \mathrm{~V}$. cholerae samples from the Yemen epidemic, and worked out each one's place in a global phylogenetic tree of more than 1,000 other isolates. They found that the strain originated in south Asia, and caused outbreaks in East Africa before spreading to Yemen. The findings are in line with an emerging picture of cholera in which south Asia serves as a cradle of new strains that lead to waves of cholera outbreaks worldwide.

Existing cholera strains in Africa are often thought to be locally entrenched. In fact, insights from genomics suggest that previous pandemic strains died out in Africa within a relatively short period the most persistent one hung around for 28 years - and that, without imports of new strains, cholera might eventually vanish from Africa altogether.

Two seminal papers on the genomics of cholera spread were published in November 2017 (a month after the WHO road map). The first traced the genomic history of a cholera pandemic that started in Indonesia in 1961 (F.-X. Weill et al. Science 358, 785-789; 2017). The scientists studied 1,070 whole-genome sequences of samples of $V$. cholerae collected in 45 African countries over almost 50 years. They discovered that all epidemics on the continent had been caused by a single expanded lineage of cholera from Asia, which had been introduced on at least 11 occasions since 1970.

In the second study, researchers investigated the whole genomes of cholera strains in Central and South America, and reached a similar conclusion: although local strains can cause sporadic cases of cholera, it is new imports of Asian strains that fuel epidemics (D. Domman et al. Science 358, 789-793; 2017).

This altered picture of disease spread revealed by genomics shows that, although the WHO rightly focuses its efforts on controlling cholera in Africa, where most cases occur, it is crucial to tackle cholera at its source in Asia to reduce outbreaks in the long term. Asia is also where most antibiotic-resistant strains of cholera emerge.

The WHO road map appropriately concentrates on the basics of cholera control. The disease, which spreads through faecal contamination of food and water, can easily be prevented by the use of basic systems for sanitation and by providing clean drinking water. Infection can cause severe, acute diarrhoea that can kill within hours, but most cases are mild and can be swiftly treated by prompt administration of an oral hydration solution.

Cholera is often a marker of extreme poverty, and of vulnerable populations afflicted by conflicts or natural disaster. It causes an estimated
1.3 million to 4 million cases every year, and results in 21,000 to 143,000 deaths worldwide. This decade has witnessed major outbreaks in Haiti, Nigeria, the Democratic Republic of the Congo, Ghana, the Horn of Africa and East Africa. The war in Yemen has caused such destruction that more than half of the population has no access to sanitation or clean water, which helps to explain why the nation had more than 1 million cholera cases and more than 2,000 people died from the disease

"Decent cholera
surveillance
demands
comprehensive
reporting of
outbrealks."
in 2017. These figures make Yemen's cholera epidemic the largest in recent history.

Genomics can complement conventional methods of surveillance and diagnosis by placing outbreaks in a global context. Given that the costs of sequencing cholera genomes have fallen over the past ten years, the WHO should consider transforming today's ad hoc genomic surveillance of cholera into a formal arrangement. This would organize sample collection and sequencing, providing information on the global emergence and circulation of cholera strains almost in real time - much as the WHO does now for strains of the influenza virus that infect humans.

Decent cholera surveillance also demands comprehensive reporting of outbreaks, and sharing of biological samples. Yet many choleraendemic countries do not count or report the number of cases. Some nations in the Horn of Africa, East Africa and elsewhere also often report a major outbreak as 'acute watery diarrhoea' without saying which pathogen caused it. Countries often fear the public's reaction or the negative economic consequences, such as falling tourism, if they declare outbreaks of the stigma-laden disease.

Yet as with many infectious diseases, the sooner control efforts are brought to bear, the easier it will be to prevent the rapid spread of an epidemic. Collecting and sharing more samples during outbreaks gives a fuller epidemiological and geographical picture, too.

A WHO stockpile of cheap and effective oral cholera vaccines is now available to countries that declare an outbreak - something that should give nations an incentive to report cases. And if a country is transparent about its cholera outbreaks, it is more likely to share samples from them. Through genomics, nations have much to gain in terms of improving understanding of cholera and being able to curb it. And that helps to build a more complete picture of the global spread and dynamics of this horrible disease.

\section{Ring cycle}

\section{Particle physics faces a controversial decision over funding of the next European supercollider.}

$\mathrm{L}$ ast week, officials at CERN outlined their vision of the European physics facility's future. Over the next two decades, they want to build a €5-billion (US\$5.7-billion), 100-kilometre-long, circular tunnel - about the length of the Washington DC Beltway. This would host the next big collider, smashing electrons and positrons, as well as a more powerful version of the existing Large Hadron Collider (LHC). The entire programme could end up costing $€ 30$ billion in construction costs alone.

CERN, near Geneva, Switzerland, certainly has the credibility to pull off such an ambitious programme. But not everyone in the research world has embraced it (see page 410 ), with some baulking at the proposed cost. In return, some in the particle-physics community acted as if such scepticism were a betrayal of science. A few took to blogs and social media to launch personal attacks against their critics.

It should go without saying that any decision on whether to fund a scientific project must weigh the costs and benefits, and whether it represents a missed opportunity to fund something else. It is essential that researchers and others debate this - and are seen to do so - in good faith and with respect for a diversity of opinions.

Some physicists think that the science case for building a bigger collider has become weaker since the LHC began operations. Its main triumph was the discovery of the Higgs boson in 2012. After the Higgs, theorists expected that the LHC would open up a world of more-massive particles, the study of which would require a new machine. No such particles have yet materialized. This has lent support to the idea that there are no new ones to be found, even in a larger collider. Consequently, the main argument for building such a machine rests on exploration.

Yet the push to higher energies is not the only way to pursue an ambitious programme. After the United States shut down the Tevatron accelerator in 2011, it moved on from building such machines. Its flagship Fermi National Accelerator Laboratory near Chicago, Illinois, has shifted focus to neutrino experiments. These do not require acceleration to the most extreme energies, but are likely to produce many key results.

CERN's proposal is its opening bid in a priority-setting process for European particle physics. Experts and policymakers will be evaluating options, and are due to announce their findings in 2020. The decisions will affect the field for the better part of a century. Whether to go for a supercollider or for cheaper options will be a difficult and inevitably controversial choice. Hopefully, it will be a decision on which rational, well-meaning people can agree to disagree. 